\title{
Sand storms: CFD analysis of Reynolds stress and collision stress of particles near sand bed
}

\author{
Yu Zhang, Jiecheng Yang, Dayou Liu*, Xiaolin Wei, Lixin Yu \\ Institute of Mechanics, Chinese Academy of Sciences, Beijing 100190, China
}

\section{A R T I C L E I N F O}

\section{Article history:}

Received 19 May 2009

Received in revised form 19 October 2009

Accepted 22 November 2009

\section{Keywords:}

Particle Reynolds stress

Collision stress

Sand bed

DEM

\begin{abstract}
A B S T R A C T
Sand storm is a serious environmental threat to humans. Sand particles are transported by saltation and suspension, causing soil erosion in one place and deposition in another. In order to prevent and predict sand storms, the causes and the manners of particle motions must be studied in detail. In this paper a standard $k-\varepsilon$ model is used for the gas phase simulation and the discrete element method (DEM) is used to predict the movements of particles using an in-house procedure. The data are summarized in an Eulerian-Eulerian regime after simulation to get the statistical particle Reynolds stress and particle collision stress. The results show that for the current case the Reynolds stress and the air shear stress predominate in the region $20-250 \mathrm{~mm}$ above the initial sand bed surface. However, in the region below $3 \mathrm{~mm}$, the collision stress must be taken into account in predicting particle movement.
\end{abstract}

(C) 2010 Chinese Society of Particuology and Institute of Process Engineering, Chinese Academy of Sciences. Published by Elsevier B.V. All rights reserved.

\section{Introduction}

Aeolian sand movement causes sand storms, desertification and other serious environmental problems. There are three modes of aeolian sand motions, i.e., creep, saltation and suspension ( $\mathrm{Li} \& \mathrm{Guo}$, 2008). Creep particles are moving by the impact of saltation particles, they are staying in contact each other, tending to creep along the surface, and cannot be lifted up by wind. Saltation particles jump to the downstream and their trajectories are determined by both air drag force and particle gravity. If a particle is transported by air in suspension, the particle will follow the air, and until the air decelerates, the particle will not settle down again. However, there is no definite standard to distinguish creeping from saltation as well as saltation from suspension. It is widely accepted that the particles are likely to be suspended by wind and collisions between particles are very rare in the region far above the sand bed. On the basis of this assumption, a numerical method was proposed to simulate particle suspension without collision (Anderson \& Haff, 1988, 1991). The numerical method requires a height to be set that can be treated as the lower boundary of the calculation domain above which particle collision does not occur. Furthermore, it is assumed that the velocities of jumping particles through the boundary can be calculated using a probability distribution function (PDF) because the velocities of jumping particles are assumed to be determined by

\footnotetext{
* Corresponding author. Tel.: +86 10 82544231; fax: +86 1062561284 .

E-mail address: dyliu@imech.ac.cn (D. Liu).
}

the velocities of impacting particles. (Anderson \& Haff, 1988, 1991; McEwan \& Willetts, 1991, 1993). Before conducting the simulation, two questions need to be answered. First, where is the boundary? Second, are the velocities of jumping particles only determined by the velocities of impacting particles? To the authors' knowledge, although new results have been reported about the velocity distribution of jumping particles (Sun, Wang, \& Xu, 2001; Zheng, Zhu, \& Xie, 2008), there are still no final answers to these two questions. And all the experimental and numerical studies have been focused on the aeolian sand movement in a tunnel, which is different from that in nature.

In this paper the $k-\varepsilon$ model is used for the simulation of gas phase turbulence and the DEM method is used to monitor particle motion and collision (Kang, Guo, \& Liu, 2008). CFD-DEM coupling method avoids all the assumptions for building a splash function. It can simultaneously give particle velocities and positions as well as the gas velocity. Although CFD-DEM coupling method has visible advantages, its calculation cost is very large. In this paper, the following assumptions are used to accelerate the simulation process:

1. The simulation is two-dimensional.

2. The diameters of the particles are assumed to be uniform and equal to $0.33 \mathrm{~mm}$, which is larger than the averaged diameter of sand particles $(0.22 \mathrm{~mm})$.

3. The simulated particles are softer than the sand particles. It saves the computational time for particle collision calculation. 
4. The friction velocity of air is $3.5 \mathrm{~m} / \mathrm{s}$ which is larger than most of the cases of reality $(0.5-2.0 \mathrm{~m} / \mathrm{s})$ to enhance particle movement. The friction velocity is used as a characteristic velocity, defined as follows (Schlichting \& Gersten, 1999):

$$
u_{*} \equiv \sqrt{\frac{\tau_{\omega}}{\rho}}
$$

where $\tau_{\omega}$ is the shear stress of air and $\rho$ is the air density.

The objective of this paper is to propose the particle stresses in an Eulerian-Eulerian framework that helps to understand the momentum transport process from the air to the sand. When aeolian sand movement takes place, in the near ground region the particle Reynolds stress and the generalized particle collision stress will play a crucial role in the momentum transport process. It is essentially different from the pure air motion, in which only air shear stress predominates in the momentum transport.

\section{CFD modeling}

\subsection{Gas phase equations}

The continuity and momentum equations of the gas phase are as follows (Kang, 2008):

$\frac{\partial}{\partial t}\left(\alpha_{f} \rho_{f}\right)+\nabla \cdot\left(\alpha_{f} \rho_{f} \boldsymbol{u}_{f}\right)=0$

$\frac{\partial}{\partial t}\left(\alpha_{f} \rho_{f} \boldsymbol{u}_{f}\right)+\nabla \cdot\left(\alpha_{f} \rho_{f} \boldsymbol{u}_{f} \boldsymbol{u}_{f}\right)=-\alpha_{f} \nabla p+\nabla \cdot\left(\alpha_{f} \boldsymbol{\tau}_{f}\right)+\alpha_{f} \rho_{f} \boldsymbol{g}-\boldsymbol{f}_{\mathrm{drag}},(2)$

where $\rho_{f}, \mathbf{u}_{f}$ and $p$ are the fluid density, velocity and pressure, respectively, $\mathbf{g}$ is gravity acceleration, $\boldsymbol{\tau}_{f}$ is the fluid shear stress, $\alpha_{f}$ is the volume fraction of fluid, and $\mathbf{f}_{\mathrm{drag}}$ is the volumetric fluid-particle interaction force. $\boldsymbol{\tau}_{f}, \alpha_{f}$ and $\mathbf{f}_{\mathrm{drag}}$ are expressed as

$\boldsymbol{\tau}_{f}=-\frac{2}{3}\left(\mu_{\mathrm{eff}} \nabla \cdot \boldsymbol{u}_{f}\right) I+\mu_{\mathrm{eff}}\left[\nabla \boldsymbol{u}_{f}+\left(\nabla \boldsymbol{u}_{f}\right)^{T}\right]$,

$\alpha_{f}=1-\sum_{k=1}^{N} \frac{V_{p k}}{\Delta V}$

$\boldsymbol{f}_{\text {drag }}=\frac{1}{\Delta V} \sum_{k=1}^{N} \boldsymbol{F}_{\mathrm{drag}, k}$,

where $\mu_{\text {eff }}$ is the fluid effective viscosity, $I$ is the unit tensor, $\Delta V$ and $V_{p k}$ are the volume of a computational cell and the volume of particle $k$ inside this cell, respectively, and $N$ is the number of particles in the cell. For the 2D flow, $\Delta V=\Delta x \Delta y d_{p}, \Delta x$ and $\Delta y$ are the lengths of a computational cell in the $x$ and $y$ directions, respectively, and $d_{p}$ is the particle diameter. $\mathbf{F}_{\mathrm{drag}}$ is the fluid drag force on a particle, which can be described as (Di Felice, 1994):

$\boldsymbol{F}_{\mathrm{drag}}=\frac{C_{d 0}}{8} \pi d_{p}^{2} \rho_{f} \alpha_{f}^{2}\left|\boldsymbol{u}_{f}-\boldsymbol{u}_{p}\right|\left(\boldsymbol{u}_{f}-\boldsymbol{u}_{p}\right) \alpha_{f}^{-\chi}$,

where $\chi=3.7-0.65 \exp \left[-\left(1.5-\log R e_{p}\right)^{2} / 2\right] . C_{d 0}, R e_{p}$ and $\mu_{f}$ are the fluid drag coefficient, the particle Reynolds number and the fluid viscosity, respectively:

$C_{d 0}=\left(0.63+\frac{4.8}{R e_{p}^{0.5}}\right)^{2}$,

$\operatorname{Re}_{p}=\frac{\alpha_{f} \rho_{f} d_{p}\left|\boldsymbol{u}_{f}-\boldsymbol{u}_{\mathrm{p}}\right|}{\mu_{f}}$,
The fluid turbulence is treated using the standard $k-\varepsilon$ turbulent model (Launder \& Spalding, 1972).

\subsection{Equations of particle motion}

The equations of translational and rotational motions of particle $k$ are expressed as

$m_{p} \frac{\mathrm{d} \boldsymbol{u}_{p k}}{\mathrm{~d} t}=m_{p} \boldsymbol{g}+\boldsymbol{F}_{\mathrm{drag}, k}+\sum_{r=1}^{N_{r}}\left(\boldsymbol{f}_{n, k r}+\boldsymbol{f}_{t, k r}\right)$,

$I_{p} \frac{\mathrm{d} \boldsymbol{\omega}_{k}}{\mathrm{~d} t}=\sum_{r=1}^{N_{r}} \boldsymbol{T}_{k r}$

where $m_{p}, \mathbf{u}_{p k}$ and $\boldsymbol{\omega}_{k}$ are the mass, translational and angular velocities of particle $k$, respectively, $\mathbf{f}_{n, k r}$ and $\mathbf{f}_{t, k r}$ are the normal and tangential forces between particle $k$ and $r$, respectively, $N_{r}$ is the number of the particles in contact with particle $k, \mathbf{T}_{k r}$ is the torque between particle $k$ and $r$, and $I_{p}$ is the moment of inertia of the particle:

$I_{p}=\frac{1}{10} m_{p} d_{p}^{2}$

\subsection{Inter-particle collision model}

The soft sphere model is used to describe inter-particle collisions. The soft sphere model can treat multiple particle contacts and provide information about the transfer of inter-particle forces.

The inter-particle forces can be described as follows (Crowe, Sommerfeld, \& Tsuji, 1997):

$\boldsymbol{f}_{n, k r}=-k_{s} \boldsymbol{\delta}_{n}-\eta v_{n, k r}$

$\boldsymbol{f}_{t, k r}=\left\{\begin{array}{l}-k_{s} \boldsymbol{\delta}_{t}-\eta v_{t, k r}, \quad\left|\boldsymbol{f}_{t, k r}\right| \leq \mu_{s}\left|\boldsymbol{f}_{n, k r}\right| \\ -\mu_{s}\left|\boldsymbol{f}_{n, k r}\right| \boldsymbol{t}, \quad\left|\boldsymbol{f}_{t, k r}\right|>\mu_{s}\left|\boldsymbol{f}_{n, k r}\right|\end{array}\right.$

where $k_{s}$ and $\eta$ are the stiffness and damping coefficient, respectively, $\mu_{s}$ is the friction coefficient; all are isotropic parameters. $\boldsymbol{\delta}$ is the displacement vector between two contacting particles, and $\mathbf{v}_{k r}$ is the relative velocity vector between two contacting particles, defined as

$$
\begin{aligned}
v_{n, k r}= & \left(v_{k r} \cdot \boldsymbol{n}\right) \boldsymbol{n}, v_{t, k r}=v_{k r}-v_{n, k r} \text { and } v_{k r}=\boldsymbol{u}_{p k}-\boldsymbol{u}_{p r} \\
& +\boldsymbol{\omega}_{k} \times \mathbf{R}_{k}-\boldsymbol{\omega}_{r} \times \mathbf{R}_{r},
\end{aligned}
$$

where $\mathbf{R}$ is a vector from the mass centre of the particle to the contact point, $\mathbf{n}$ is the unit vector from the centre of particle $k$ to that of particle $r$ and $\mathbf{t}$ is the unit tangential vector:

$\boldsymbol{n}=\frac{\boldsymbol{R}_{k}}{\left|\boldsymbol{R}_{k}\right|}$ and $\boldsymbol{t}=\frac{v_{t, k r}}{\left|v_{t, k r}\right|}$.

\subsection{Reynolds stress and generalized collision stress}

\subsubsection{Reynolds stress}

Suppose the velocity in the direction $x_{i}(i=1,2,3)$ of particle $k$ is $u_{p k, i}$, After numerical simulation, the particle mean velocities $\bar{u}_{p, i}$ in the direction $x_{i}(i=1,2,3)$ and the particle Reynolds stress $R_{i j}(i$, $j=1,2,3$ ) can be directly derived as follows:

$\bar{u}_{p, i}=\frac{\sum_{k=1}^{N} u_{p k, i}}{N}$

$\overline{u_{p, i}^{\prime} u_{p, j}^{\prime}}=\frac{\sum_{k=1}^{N}\left(u_{p k, i}-\bar{u}_{p, i}\right)\left(u_{p k, j}-\bar{u}_{p, j}\right)}{N}$, 


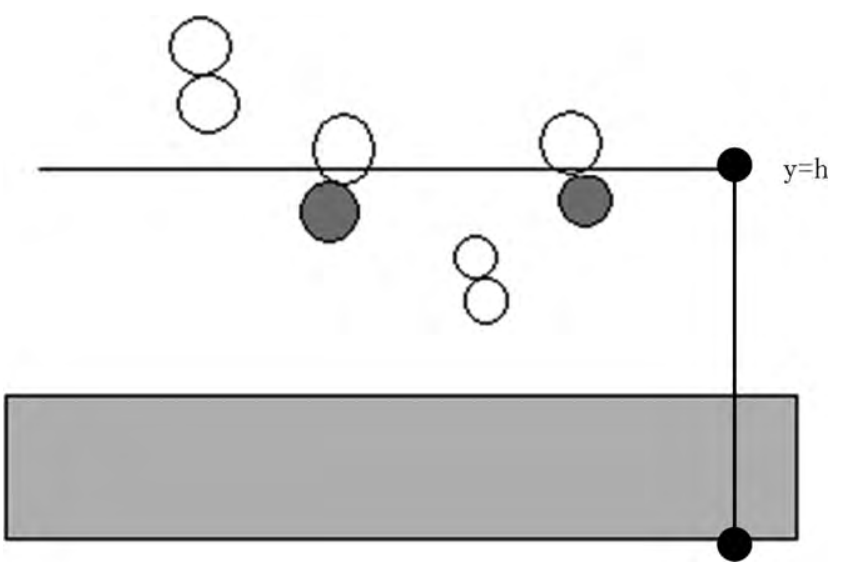

Fig. 1. The criteria for selecting particle collisions.

$R_{i j}=-\rho_{m} \overline{u_{p, i}^{\prime} u_{p, j}^{\prime}}$,

where $\rho_{m}$ is the mean density of particles. $u_{p i}$ and $u_{p j}$ are the particle velocity components in horizontal and vertical directions. The particle Reynolds stress $\left(R_{i j}\right)$ is caused by the particle momentum exchange at a surface where particles flow in and out.

\subsubsection{Generalized collision stress}

Fig. 1 illustrates the criteria for selecting particles that are likely to collide with each other at the level $h$ above the sand bed. When a collision occurs, the collision force for each particle is recorded. For the collisions considered, one particle must be below the level $h$ whereas the other must be above the level h. Only those particles whose distance from the level $h$ is less than particle diameter, like those filled in grey in Fig. 1, need to be considered for calculation. The area averaged collision force that is applied on those particles is defined as the generalized collision stress which is expressed as

$F_{c}=\frac{\sum_{k=1}^{n} f_{x}}{A}$.

where $f_{X}$ is the $x$ component of the collision force for a individual particle. $A$ is the surface area.

\section{Numerical method and simulated conditions}

The equations for the gas phase are solved by the conventional SIMPLEC method. The finite volume method is applied to discretize the gas equations on non-staggered rectangular grids. The secondorder central difference scheme is used for the diffusion terms. The QUICK scheme is used for the convective terms of the momentum equations. The motion equations of discrete particles are solved by the explicit time integration method. In order to reduce the CPU time, for each particle, the neighbour list is used to store all neighbours, and a check for possible collisions is performed only for the particles in this list. The computational domain is a $2 \mathrm{D}$ rectangular region of $0.35 \mathrm{~m}$ high and $0.15 \mathrm{~m}$ wide, which is shown in Fig. 2. The periodic boundary condition is used for the inlet and the outlet. When a particle leaves from the inlet or the outlet, it will enter via the other port. A constant shear stress of $14.7 \mathrm{~Pa}$ is applied on the top surface which corresponds to a friction velocity of $3.5 \mathrm{~m} / \mathrm{s}$. In CFD calculations, the product of the effective viscous coefficient of turbulence and the velocity gradient at the top surface should always keep constant at $14.7 \mathrm{~Pa}$.

Initially, 11,000 particles are uniformly distributed in the calculation domain and start to fall down to the bottom. After particle deposition a sand bed is automatically formed with a thickness of $3.6 \mathrm{~mm}$. In the sequent discussions, the height is defined as the

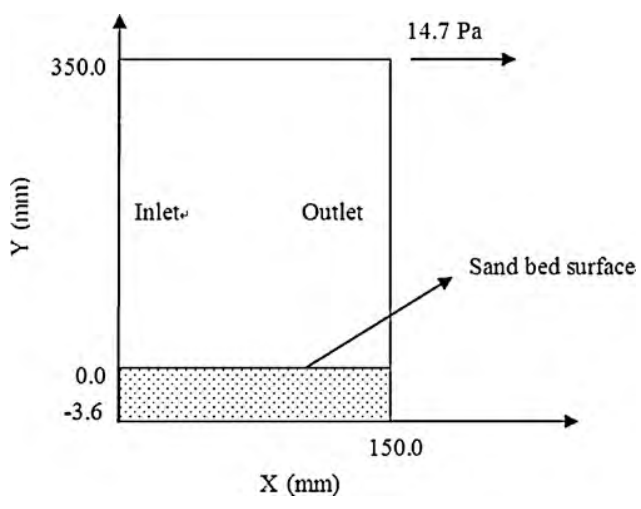

Fig. 2. Calculation domain.

vertical distance from the sand bed surface. Particles impacting the sand bed are used to initiate the motion of sand particles on the surface of the sand bed. The bottom of the sand bed is treated as a fixed surface. All particles below that surface are assumed to be at rest at all times and are not included in the calculation domain.

The simulated data in the steady state are carefully studied. The steady state means that the statistically averaged flow parameters do not change with time. In practical calculations the sand transport rate per unit width is monitored. When the aeolian sand movement reaches the steady state, the sand transport rate per unit width will be a constant value.

In simulation the density of gas is $1.2 \mathrm{~kg} / \mathrm{m}^{3}$, dynamic viscosity $1.785 \times 10^{-5}$ Pa s, particle diameter $0.33 \mathrm{~mm}$ and particle density $2650 \mathrm{~kg} / \mathrm{m}^{3}$. The friction coefficient is 0.4 , the stiffness coefficient $1500 \mathrm{~N} / \mathrm{m}$, and the damping coefficient 0.002 . The restitution coefficient is 0.85 , which is determined by the damping and stiffness coefficients as (Kang, 2008)

$e=\exp \left(-\frac{\eta \pi}{\sqrt{4 m_{\mathrm{p}} k_{\mathrm{s}}-\eta^{2}}}\right)$.

The computational time-step for the fluid is chosen as $2.0 \times 10^{-5} \mathrm{~s}$, and the time-step for particles is $2.0 \times 10^{-6} \mathrm{~s}$; i.e., there are 10 integration steps for the particle trajectory in every time-step for fluid motion.

\section{Results and discussions}

\subsection{Collision frequency}

It should be pointed out that some particles are accumulated at the bottom of the calculation domain and contact each other all the time. It is hard to judge whether this kind of contact is a collision; however, this sort of particle contact is treated as a "long term collision" for the convenience of statistical work in the following discussion, and the collision stress is named "generalized collision stress".

Fig. 3 shows the calculated incidence of particle collision at different heights in the calculation domain. This result is an average over 9 times of sample collections. There are more than 2000 collisions in the region below the height $-1.0 \mathrm{~mm}$ (accumulation zone). It can be seen from this figure that the number of collisions decreases sharply as the height increases. At the height around $4 \mathrm{~mm}$ above the surface of the sand bed, there are almost no particle collisions. This result verifies the existence of a boundary, above which particle collision can be ignored. However, there is still a thin layer below that boundary and above the accumulation zone $(-0.5-3.0 \mathrm{~mm})$. 


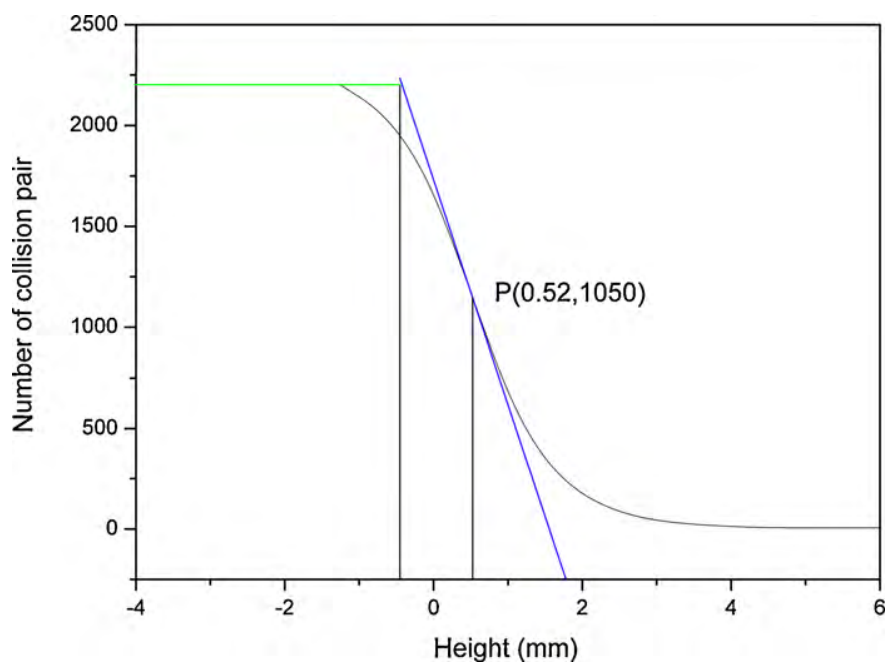

Fig. 3. Collision frequency.

The impacting and the jumping particles within this layer are defined by the direction of their vertical velocity component. For an impacting particle, the vertical velocity should be downward whereas for a jumping particle, its vertical velocity is upward, so that both impacting and jumping particles go through this layer, and the collisions occurring within this layer are totally random. Therefore, the assumption that the velocity of a jumping particle is determined only by the velocity of the impacting particle is not accurate. The assumption is acceptable only when the layer is thin enough.

\subsection{Correlation of particle velocity fluctuations}

Fig. 4 shows the correlation between particle velocity fluctuations in the $x$ and $y$ directions $\left(\overline{u_{p}^{\prime} v_{p}^{\prime}}\right)$, i.e. $\overline{u_{p, i}^{\prime} u_{p, j}^{\prime}}$ in the Eq. (14). Fig. 4(a) shows that when the height of the calculation domain is divided into 10 bins, along the height of the domain the absolute value of correlations between velocity fluctuations in the $x$ and $y$ directions first increases and then decreases. The peak value appears at a position around $100-150 \mathrm{~mm}$ height, because the bottom particle movement is restricted by collisions, and the top particles are carried by the air. Only at the middle position of the domain particles move freely and therefore the velocity fluctuations are strongest.

The similar pattern is shown in Fig. 4(b), demonstrating that when the domain is divided into 100 bins in the $y$ direction, the correlation results are fluctuated, because the number of samples in one-hundredth of the height is much less than that in one-tenth of the height; therefore, the statistical error for the space of onehundredth of the height is much greater. Even so, it can be seen that the regression curve in Fig. 4(b) is similar to that in Fig. 4(a).

\subsection{Correlation coefficient}

The correlation coefficient of particle velocities, which represents the relevant relationship between particle velocity fluctuations in different directions, is calculated by the following equation:

$C=\frac{\overline{u_{p}^{\prime} v_{p}^{\prime}}}{\sqrt{\overline{{u_{p}^{\prime} v_{p}^{\prime}}^{2}}}}$

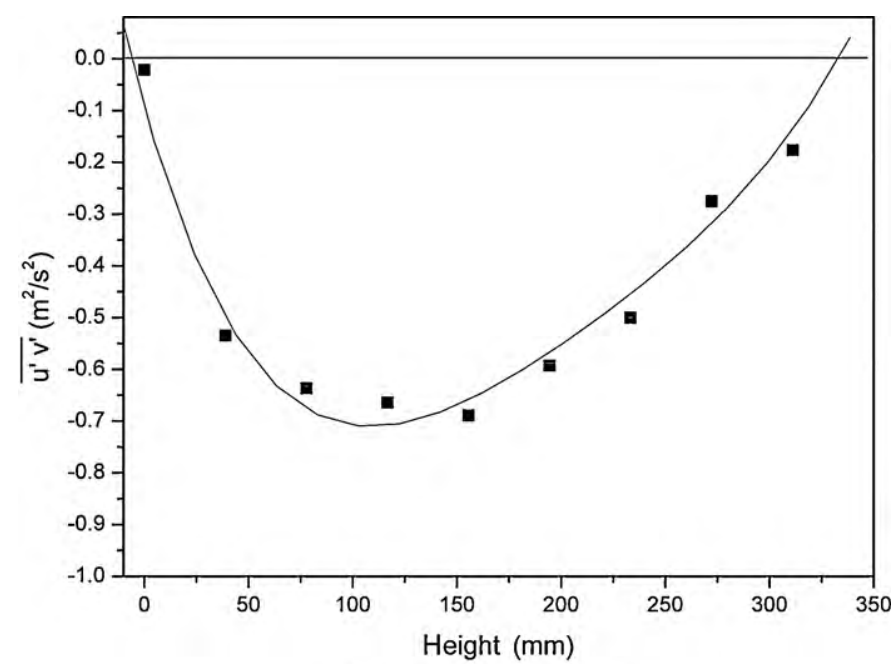

(a) 10 spaces in y direction

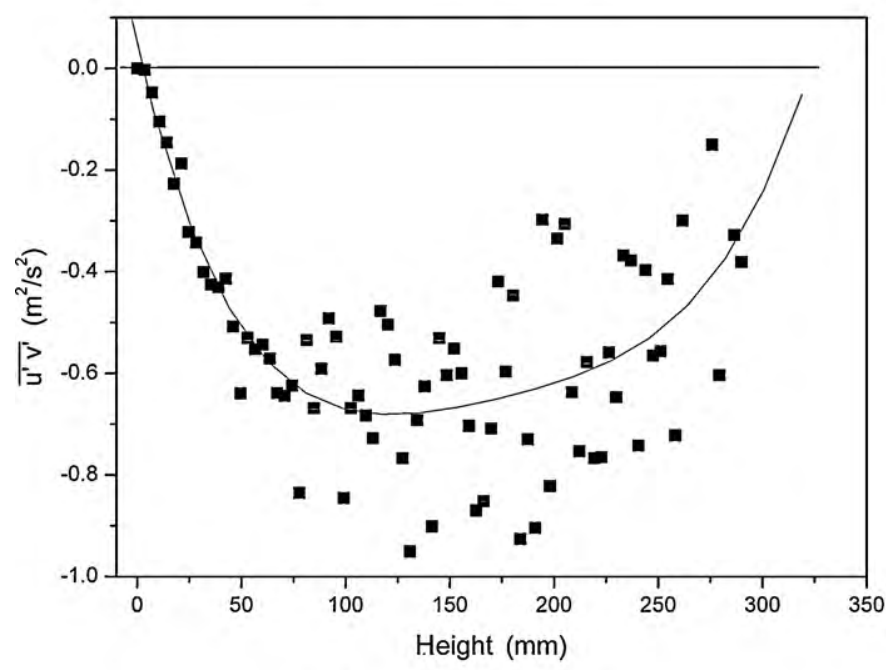

(b) 100 spaces in y direction

Fig. 4. Correlations of particle velocity fluctuations: (a) 10 spaces in $y$ direction. (b) 100 spaces in $y$ direction.

The correlation coefficient of particle velocities in both the $x$ and $y$ directions is shown in Fig. 5, indicating that as the height increases the absolute value of the correlation coefficient also increases, which means that the particle velocities in the $x$ and $y$ directions are more relevant to each other at higher region above the sand bed. This result can be illustrated by particle collisions in the region near the sand bed, where the particles collide frequently with almost no relationship between particle velocities in different directions. Since particles are accumulated at the bottom boundary of the calculation region, the correlation coefficient should be zero there. On the contrary, since particle collisions occur rarely far above the sand bed, particle velocity fluctuations in different directions are strongly relevant.

\subsection{Reynolds stress}

Fig. 6 displays the Reynolds stress of the particle phase along the height of the calculation domain, showing that the particle Reynolds stress decreases with increasing height; at the region about $250 \mathrm{~mm}$ above the bottom of the sand bed, the particle Reynolds stress approaches zero. The results imply that the par- 


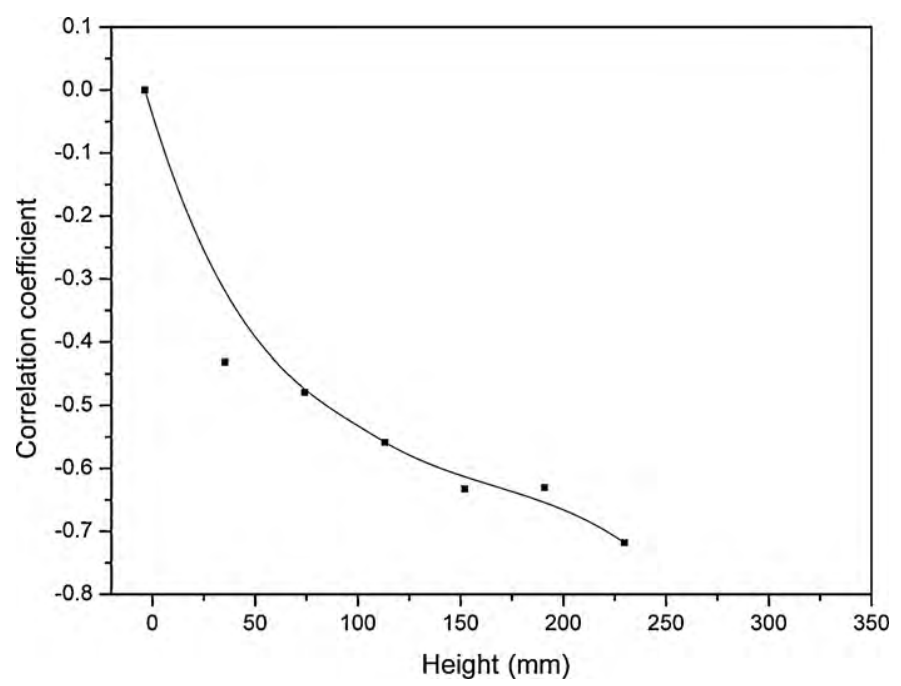

Fig. 5. Correlation coefficient of particle velocities.

ticle Reynolds stress plays a role only when the height above the sand bed is less than about $250 \mathrm{~mm}$.

It is worth noting that the Reynolds stress should be zero at the bottom of the calculation domain, as the particle velocity fluctuations must be zero there. When the height is above the accumulation zone, the Reynolds stress increases sharply and reaches a peak value within a few millimetres. However, because the CFD grids are not fine enough, it is difficult to obtain detailed information about the increase of the Reynolds stress in this region.

\subsection{Shear stress of air and air drag force}

The shear stress of air caused by the air turbulence is calculated by the following equation:

$\tau_{f, y x}=\mu_{\mathrm{eff}} \frac{\partial u}{\partial y}$,

where $\tau$ is the shear stress of air and $\mu_{\text {eff }}$ is the air effective viscosity coefficient.

Fig. 7 shows the variation of shear stress of air along the height of calculation domain. As the height increases, the shear stress of air increases. When the height is over about $250 \mathrm{~mm}$, the shear stress of air is $14.7 \mathrm{~Pa}$ which is consistent with the given shear stress at

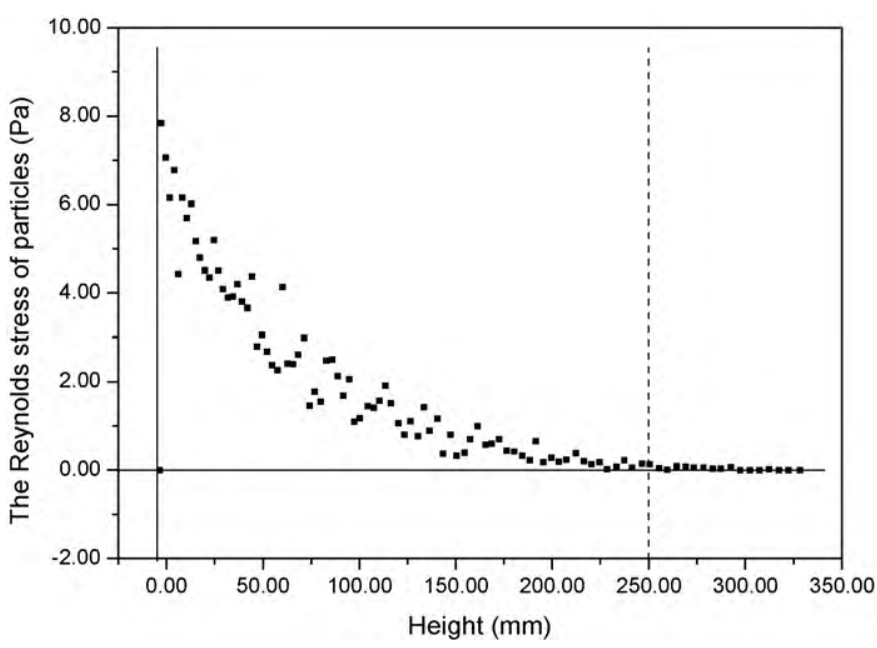

Fig. 6. Particle Reynolds stress.

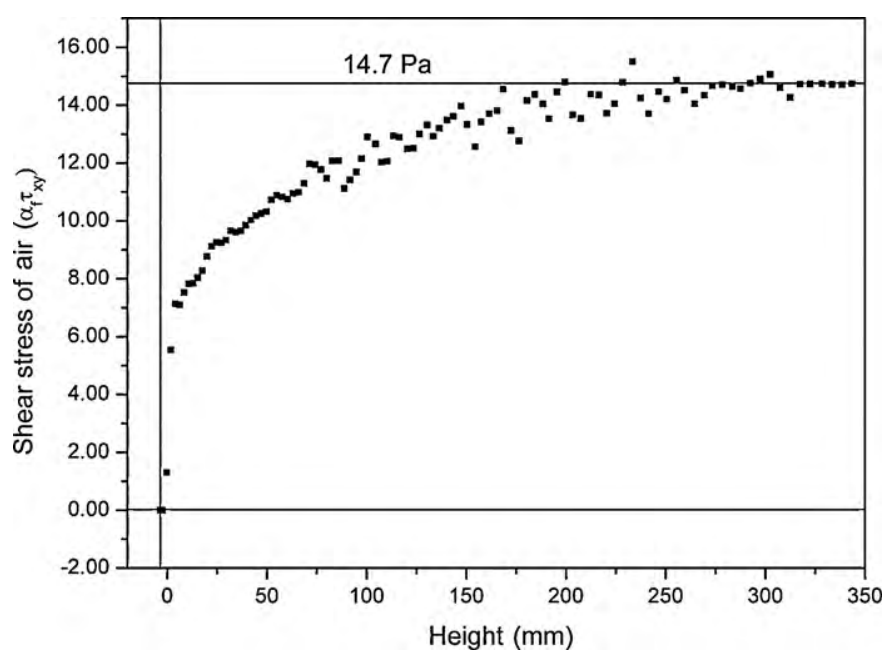

Fig. 7. Shear stress of air.

the top boundary of the calculation domain. The top boundary is assumed to be located in the main airflow, and the shear stress here should always be $14.7 \mathrm{~Pa}$. It can be seen from Fig. 7 that the main airflow region starts from about $250 \mathrm{~mm}$.

It should be pointed out that for the case discussed here there is no pressure gradient in the flow field. When the flow reaches steady state, the momentum conservation equation (Eq. (2)) in the $x$ direction can be simplified as follows:

$\frac{\partial\left(\alpha_{f} \tau_{f, y x}\right)}{\partial y}=f_{\text {drag }, x}$.

That is, when the flow reaches steady state, the air shear stress gradient is equal to the volumetric air drag force, as shown in Fig. 7, that is, the air drag force plays a role within $250 \mathrm{~mm}$ above the initial surface of the sand bed. However, if the particles are suspended above $250 \mathrm{~mm}$, they should still be driven by air drag force although the number density of particles there is very small.

\subsection{The sum of shear stress of air and particle Reynolds stress}

The sum of the shear stress of air and the particles Reynolds stress is illustrated by Fig. 8, showing that when the height above the sand bed bottom is over $20 \mathrm{~mm}$, the total stress is close to

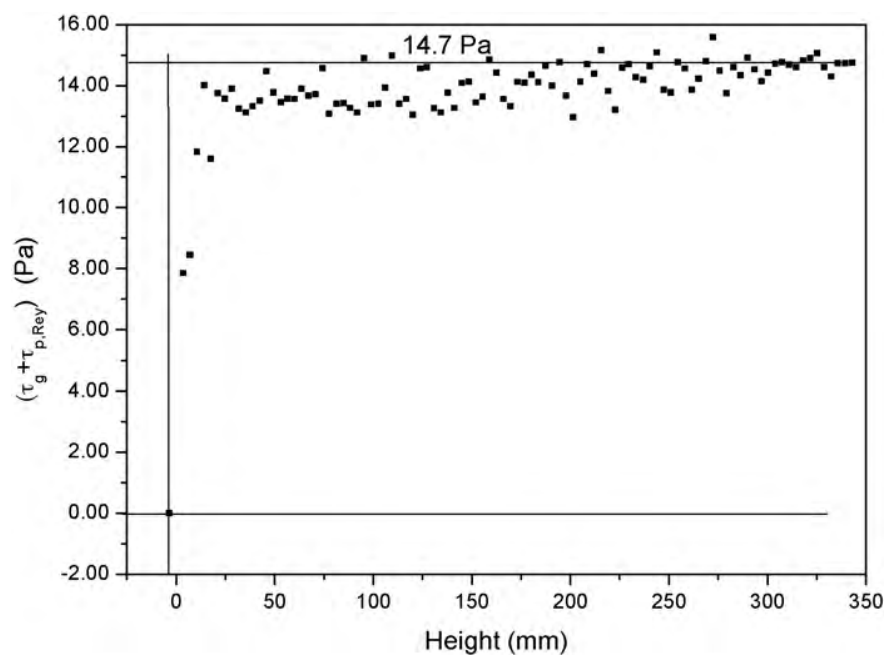

Fig. 8. Sum of shear stress of air and particle Reynolds stress. 


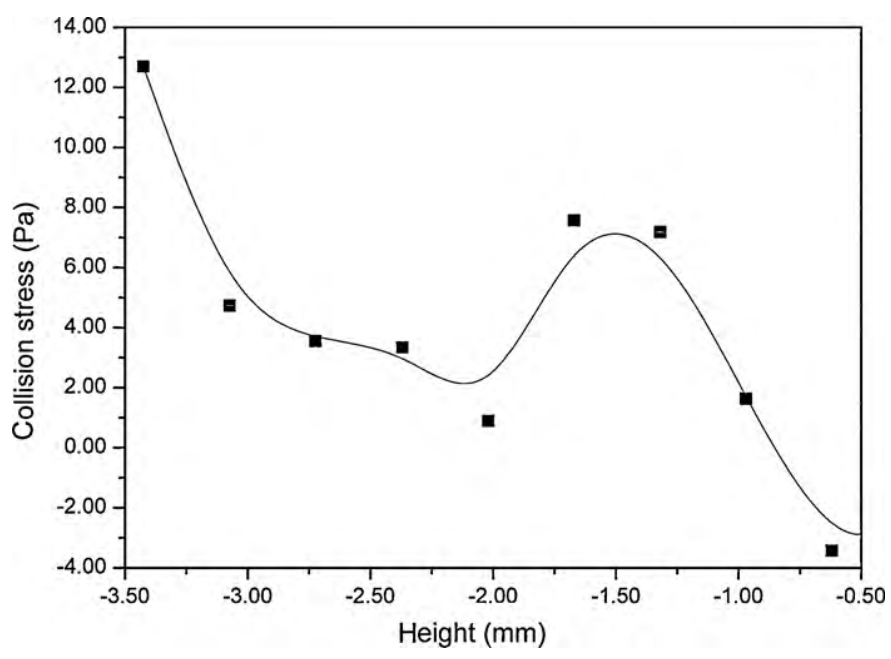

Fig. 9. Generalized particle collision stress from 3 to $6.58 \mathrm{~s}$.

14.7 $\mathrm{Pa}$, implying that the total stress for the air and particle mixture should be constant everywhere in the flow field. However, for the height below $20 \mathrm{~mm}$ above the surface of the sand bed, the total stress decreases toward the sand bed. The results imply the existence of another kind of stress taking effect in the region close to the sand bed.

\subsection{Generalized collision force of particles}

Fig. 9 shows the non-monotonic variation of the generalized collision stress with height below $-0.5 \mathrm{~mm}$ and within $3-6.58 \mathrm{~s}$. At the bottom of the sand bed, the collision force is $13 \mathrm{~Pa}$. As height increases, the collision force first decreases very quickly, and then fluctuates with height while the height is above $-2 \mathrm{~mm}$. This nonmonotonic variation cannot be attributed to lack of samples since the value at every point in the figure is an average of more than 500 samples, implying that the number of samples collected for calculating collision force at any position, must be greater than 500 .

Fig. 10 shows the variation of the generalized collision stress with height below $-0.5 \mathrm{~mm}$ within $3-8.98 \mathrm{~s}$. The shape of the curve is similar to that in Fig. 9 while the fluctuation amplitude is much smaller. These results imply that the collision force fluctuations are induced by the long time period of particle movement near the

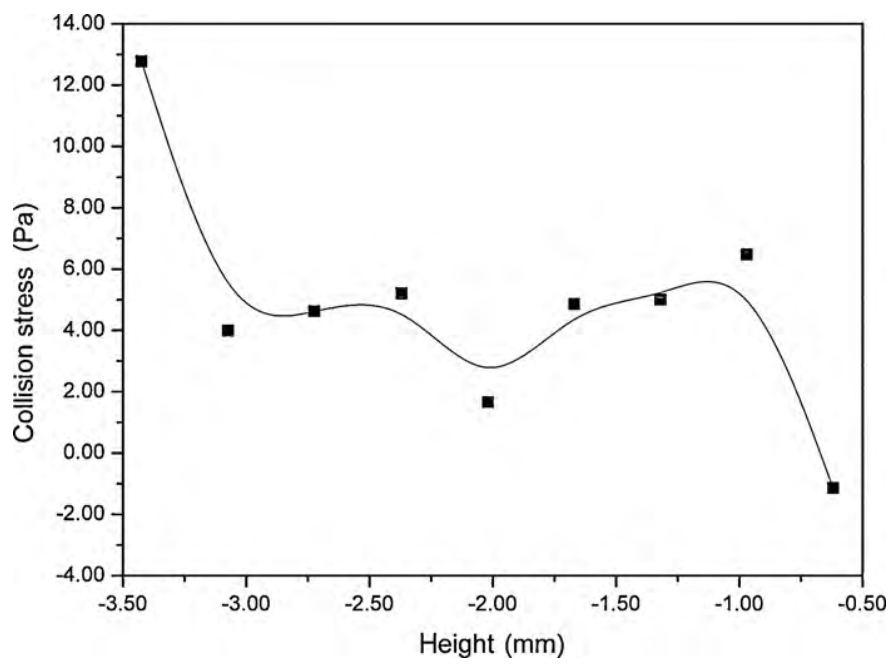

Fig. 10. Generalized particle collision stress from 3 to $8.98 \mathrm{~s}$.

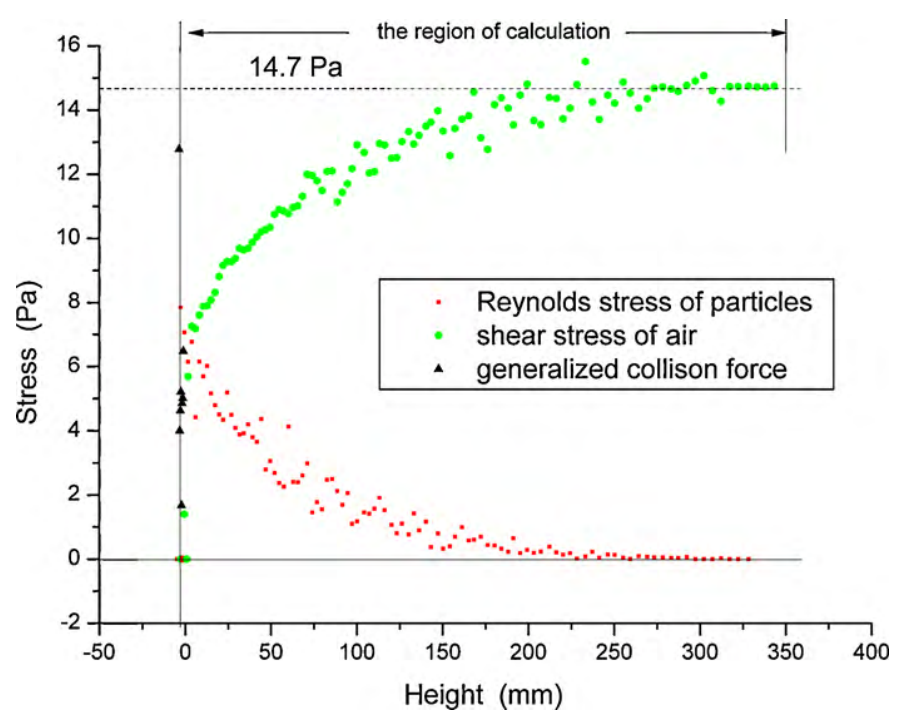

Fig. 11. Three kinds of stress.

bottom of the sand bed, and this period should be much longer than $6.58 \mathrm{~s}$ and even as long as $8.98 \mathrm{~s}$. When the time period used in the statistical analysis is shorter than the physical time period of particle movements, the results will have unavoidable fluctuations, and the analysis for longer time period will reduce the fluctuations. In conclusion, the collision force affects the movements of particles in the region below sand bed surface. Excluding the bottom of the sand bed, the averaged collision force of particles in this region is 4-5 Pa. Fig. 11 shows the distributions of the air shear stress, the particle Reynolds stress and the generalized collision force along the height, showing that the generalized collision force of particles exists only in the region very close to the bottom of the sand bed.

It should be pointed out that there are very few particle samples for measuring the collision force or Reynolds stress in the region $0-20 \mathrm{~mm}$. In order to get enough samples in that region, the total number of particle samples in the whole field must be increased. This issue will be addressed in our future work.

\subsection{Sand transport rate}

Fig. 12 gives the variation of the predicted sand transport rates with the height, showing that the sand transport rate decreases

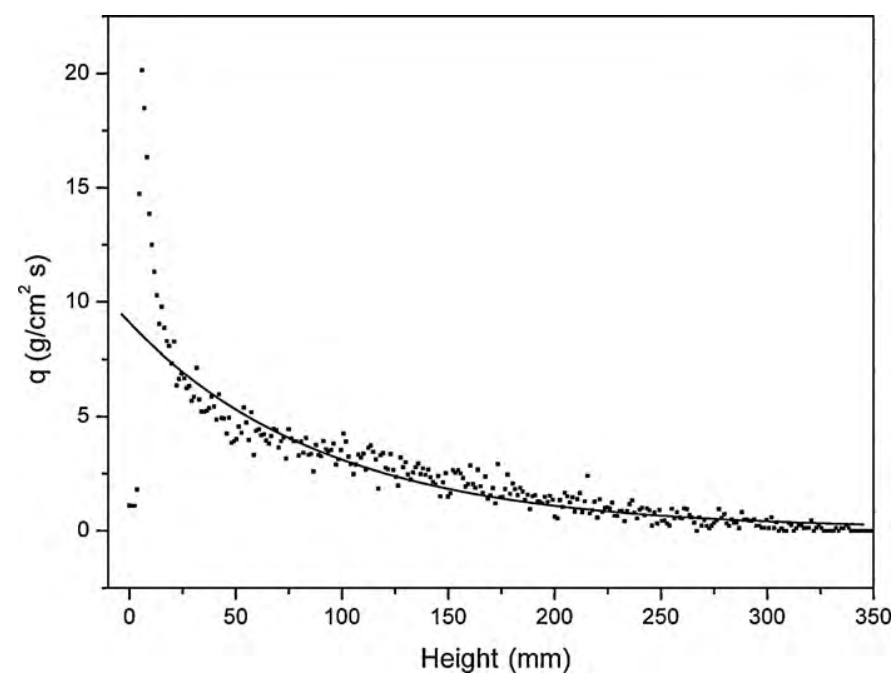

Fig. 12. Sand transport rates. 
exponentially in most of the regions. However, in the sand bed, the sand transport rate is higher than that calculated by the exponential function (the solid line) as was observed by Butterfield in his experimental work (Butterfield, 1999). The reason is that in the sand bed, the sand particles creep slowly rather than move, though creeping implies large transport rate. Moreover, the simulated sand transport rate in this paper is much higher than that observed because the calculation was two-dimensional and the air friction velocity was overestimated.

\section{Conclusion}

A CFD-DEM method is proposed for the simulation of aeolian sand movement. The proposed method avoids the assumptions required for building a splash function and gives air velocity and particle velocity simultaneously. This paper also discusses the stresses that drive the aeolian sand movement including the particle Reynolds stress, the generalized particle collision stress and the air shear stress. The discussion helps to understand both particle performance and momentum transport process in aeolian sand movement.

The specific case discussed in this paper yields the following conclusions:

- In the simulation described above, particle collisions can be ignored in the region $4 \mathrm{~mm}$ above the surface of the sand bed. This result verifies the existence of a boundary for particles, above which particle collisions can be ignored. However, there still exists a thin layer below that boundary and above the accumulation zone $(-0.5-3 \mathrm{~mm}$ in the simulated case). Both impacting and jumping particles pass through this layer, and the particle collisions within this layer are totally random. Therefore, the assumption that the velocity of a jumping particle is determined by the velocity of the impacting particle does not hold. The assumption is acceptable only when the layer is thin enough.

- Both the Reynolds stress and the air drag force control the movements of particles in the region $20-250 \mathrm{~mm}$ above the bottom of the sand bed. As the height increases, the air drag force plays a more important role and the Reynolds stress decreases rapidly.

- Only the air shear stress needs to be considered in the region $250 \mathrm{~mm}$ above the bottom of the sand bed. The suspended particles in this region are driven only by the air drag force.

- Particle collision stress affects particle movement and must be considered in the region below the surface of the sand bed.
- No data are available in this study for collision force or Reynolds stress of particles in the region $0-20 \mathrm{~mm}$ above the bottom of the sand bed. To acquire such data, the total number of particle samples needs to be increased.

- The present simulation is two-dimensional; the simulated particles are softer than the sand particles; the air shear stress in simulation is greater than most of the shear stress in reality. Therefore the simulation still needs to be improved in the future.

\section{Acknowledgments}

This work is supported by the National Natural Science Foundation of China (Grant No. 10532030) and the CAS Innovation Program. Special thanks are due to Dr. Liqiang Kang for the basic coding work.

\section{References}

Anderson, R. S., \& Haff, P. K. (1988). Simulation of eolian saltation. Science, 241, $820-823$.

Anderson, R. S., \& Haff, P. K. (1991). Wind modification and bed response during saltation of sand in air. Acta Mechanica, 1(Suppl.), 21-51.

Butterfield, G. R. (1999). Near-bed mass flux profiles in aeolian sand transport: High-resolution measurements in a wind tunnel. Earth Surface Processes and Landforms, 24, 393-412.

Crowe, C. T., Sommerfeld, M., \& Tsuji, Y. (1997). Multiphase flows with droplets and particles. Boca Raton: CRC Press.

Di Felice, R. (1994). The voidage function for fluid-particle interaction systems. International Journal of Multiphase Flow, 20, 153-159.

Kang, L. Q. (2008). Numerical simulation and theoretical analysis of aeolian sand transport. Postdoctoral Research Report, Institute of Mechanics, Chinese Academy of Sciences, China. pp. 46-47.

Kang, L., Guo, L., \& Liu, D. (2008). Reconstructing the vertical distribution of the aeolian saltation mass flux based on the probability distribution of lift-off velocity. Geomorphology, 96, 1-15.

Launder, B. E., \& Spalding, D. B. (1972). Mathematical models of turbulence. London: Academic Press.

Li, Y., \& Guo, Y. (2008). Numerical simulation of aeolian dusty sand transport in a marginal desert region at the early entrainment stage. Geomorphology, 100, 335-344.

McEwan, I. K., \& Willetts, B. B. (1991). Numerical model of the saltation cloud. Acta Mechanica, 1(Suppl.), 53-66.

McEwan, I. K., \& Willetts, B. B. (1993). Adaptation of the near-surface wind to the development of sand transport. Journal of Fluid Mechanics, 252, 99-105.

Schlichting, H., \& Gersten, K. (1999). Boundary layer theory (8th ed.). Berlin: SpringerVerlag., pp. 564

Sun, Q., Wang, G., \& Xu, Y. (2001). DEM applications to Aeolian sediment transport and impact process in saltation. Particle Science and Technology, 19, 339-353.

Zheng, X., Zhu, W., \& Xie, L. (2008). A probability density function of liftoff velocities in mixed-size wind sand flux. Science in China Series G: Physics, Mechanics and Astronomy, 51(8), 976-985. 